\title{
Peak-to-average power ratio of a Zipper signal
}

\author{
A. D. S. Jayalath and C. Tellambura \\ Faculty of Information Technology \\ Monash University, Clayton Victoria 3168, Australia \\ Email: chintha@dgs.monash.edu.au
}

\begin{abstract}
In this paper we investigate the peakto-average power ratio (PAR) and its effects on the performance of Zipper-VDSL systems. The spectrum and the out of band power when passing through a nonlinear device is also investigated. We further evaluate the performance with a recently proposed PAR reduction technique for orthogonal frequency division multiplexing (OFDM). PAR reduction of Zipper-VDSL minimizes the distortion and out of band power of the signal, reducing the dynamic range of transceiver components and nearend cross talk NEXT.
\end{abstract}

Key words - DMT, Zipper, Peak-to-average power ratio.

\section{INTRODUCTION}

Very high speed digital subscriber lines (VDSL) $[1,2]$ are capable of handling bit rates up to 52 $\mathrm{Mb} / \mathrm{s}$ over ordinary phone lines, thus giving broadband data services to customers without incurring the very high infrastructure installation costs, such as optical fiber installation and expensive wireless access. VDSL is a cost effective solution to those requirements. It can provide real broadband data services as well as competitive television services over ordinary phone lines. The range of the VDSL over copper wire is around $1.5 \mathrm{Km}$. Therefore, VDSL is primarily used to connect optical network units (cabinets) to the customer premises.

Near-end crosstalk (NEXT) and near-end echoes are some of the significant problems VDSL systems have to overcome. The Zipper duplex method [3] avoids these by using different orthogonal subcarriers in the upstream and downstream directions. Subcarriers provide frequency division duplexing (FDD) so that devices can transmit and receive data simultaneously. Although Zipper is based on ordinary discrete multitone (DMT) modulation, it has two major differences. It uses different subcarriers in different transmission directions and it adds a cyclic suffix (CS) to ensure orthogonality between the transmitted and received sig- nals. The cyclic suffix is constructed by adding a portion of the beginning of the symbol to the end of the symbol. However, It also has a cyclic prefix as in the case of ordinary DMT. The cyclic prefix is constructed by adding a portion of the end of each symbol to the beginning of the symbol. This acts as a guard interval between each symbol and prevents inter symbol interference. At the receiving end the cyclic prefix is removed and the signal is demodulated using a discrete fourier transform. Because of its construction the cyclic prefix also avoids inter carrier interference by maintaining orthogonality between the sub carriers. NEXT is further reduced by using pulse shaping at the transmitting end [4]. The length of the cyclic suffixes are increased and then these additions are pulse shaped by a gradual decrease in transmitter power. This decreases the number of discontinuities which exist in the signal between adjacent symbols. This method has the advantage that the original symbol is not affected while suppressing the sidelobes caused by a rectangular pulse-shape.

A typical Zipper signal is depicted in Figure 1. In this example Zipper symbol, alternative subcarriers are used to upstream and the down stream directions. The upstream and downstream subcarrier assignment can be changed dynamically, depending on the data rate requirement of the user. For example, when the user connect to the internet for browsing, he needs higher bandwidth for the downstream and low bandwidth for the upstream. In this situation we can assign more subcarriers for the downstream. These dynamic assignments of subcarriers are quite simple in the case of DMT based Zipper compared to the traditional FDD systems, where analog filters to isolate each channel is very complex to set up and change adaptively.

Another advantage of Zipper duplexing method is that it allows VDSL systems to co-exist with other DSL technologies such as ADSL. To prevent NEXT with ADSL systems operators can choose to 
have Zipper VDSL systems follow the ADSL duplexing scheme in the frequencies that they share (transmit in the same direction) while using VDSL frequencies above the ADSL spectrum in an arbitrary manner [3]. Zipper can also avoid channels which might cause interferences to other systems and channels severely degraded by interferences of other systems. Significant amount of radiation is caused by the drop cable connecting the distribution box to the home. This could cause RF interferences to other systems. On the other hand other systems like HAM radio may also interfere the VDSL systems. Zipper can be programmed to cancel out these effected channels.

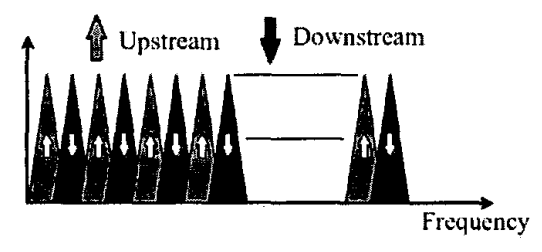

Fig. 1. Zipper signal.

The principal drawback of DMT based VDSL signal is that the peak transmitted power can be substantially larger than the average power. This high PAR causes distortion of the desired signal and increases the out of band power when passing through non linear devices and asked for larger dynamic ranges for transceiver equipment. Solutions to the PAR problem of OFDM have been proposed by several researchers $[5,6]$. In this paper peak-to-average power ratio (PAR) and the effect of non-linearity on the Zipper signal is investigated. Performance of PAR reduced Zipper signal is also evaluated through simulations. Permutation of data sequences are used to to reduce the high PAR. PAR reduced Zipper signals minimizes the inband distortion and out of band power. Therefore, PAR reduction will help to reduce the NEXT and the dynamic operating range of the transceiver equipment at the price of a small amount of overhead per symbol and the system complexity.

This paper is organized as follows. Next section describes the Zipper-VDSL system model. Section 3 describes the nonlinearity device used to observe the out of band power. Simulation and theoretical results are presented in Section 4 and Section 5 concludes the paper.

\section{SYSTEM DESCRIPTION}

Zipper signals use different subcarriers in upstream and down stream directions. Let, the indices of the upstream subcarriers be $I_{i, u p}, 0 \leq I_{i, u p} \leq$ $2 N$. Where, $N$ is the total number of subcarriers. Since the allocation of the upstream and downstream subcarriers can be changed depending on the user's requirement, $I_{i, u p}$ are not fixed values. The upstream Zipper signal can then be expressed as [3],

$x(t)= \begin{cases}\sum_{n \in I_{i, u p}} X_{n} e^{\frac{j 2 \pi n \Delta f t}{2 N}} & t \in\left[0, \frac{2 N+C P+C S}{\Delta f}\right], \\ 0 & \text { otherwise. }\end{cases}$

Where $C P$ is the length (in samples) of the cyclic prefix, $C S$ is the length of the cyclic suffix, and $\Delta f$ is the sampling frequency. Since $x(t)$ is a real-valued signal, the data $X_{n}$ must satisfy

$$
X_{n}= \begin{cases}\operatorname{real}\left(X_{n}\right) & n=0 \\ X_{2 N-n}^{*} & N>n>2 N \\ \text { imaginary }\left(X_{n}\right) & n=N\end{cases}
$$

where the asterisk denote the complex conjugate. Same set of equations can be written for the downstream Zipper signal. The indices of the down stream subcarriers are $I_{i, d o w n}, 0 \leq I_{i, \text { down }} \leq$ $2 N$.The downstream part of the Zipper frame is subset of the complement set to $I_{i, u p}\left(I_{i, \text { down }} \subseteq\right.$ $\left.\{0,1, \ldots, 2 N-1\} \backslash I_{i, u p}\right)$.

The PAR of the Zipper signal in (1) can be defined as

$$
\mathrm{PAR}=\frac{\max |x(t)|^{2}}{E\left[|x(t)|^{2}\right]}
$$

where $E[x]$ is the expected value of $x$. The PAR of the continuous-time OFDM signal cannot be computed precisely by the use of the Nyquist sampling rate [7], which amounts to samples per symbol. In this case, signal peaks are missed and PAR reduction estimates are unduly optimistic. Oversampling by a factor of 4 is sufficiently accurate and is achieved by the simple computation of the $4 N$ point zero-padded IDFT of the data frame. Herein we shall assume oversampling by a factor of 4 for all further calculations.

A simple permutation technique is proposed to reduce the PAR of the signal [8]. In this approach $K-1$ interleavers are used at the transmitter. These interleavers produce $K-1$ permuted frames of the input data before mapping into transmit symbols. 
The four times oversampled IDFT of each frame (including the uncoded frame) is used to compute its PAR. The minimum PAR frame of all the $K$ frames is selected for transmission. The identity of the corresponding interleaver is also sent to the receiver as side information. Figure 2 depicts the DMT based Zipper-VDSL transmitter with data permutations to reduce the PAR.

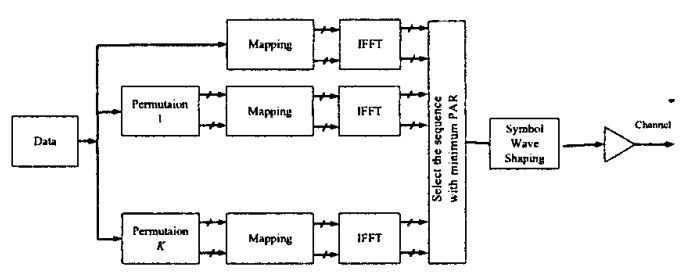

Fig. 2. System block diagram.

\section{NONLINEAR TRANSMITTER CHARACTERISTICS}

The VDSL signals are subject to various hardware non-linearities in both the transmitter and receiver. These include signal clipping in the ana$\log$ to digital (A/D) converter and signal clipping in the IFFT and FFT processors with a limited word length. Such non-linearities not only affect the performance of an VDSL system, but also may affect the system performance of an adjacent channel, causing severe NEXT. The out-of-band power increases if enough dynamic ranges are not provided at the transmitter equipment. The simplest form of non-linearity is the soft limiter.

\section{A. Soft limiter (SL)}

Since the amplitude modulation to phase modulation (AM/PM) component is zero the nonlinear characteristics of a SL can be written as

$$
g(x)= \begin{cases}x & |x| \leq A, \\ A e^{j \varphi} & |x|>A,\end{cases}
$$

Where $x$ is the input to the SL, $A$ is the saturated output and $\varphi$ is the phase angle of the input $x$. Although most physical components will not exhibit this piecewise linear behavior, the SL can be a good model if the nonlinear element is linearized by a suitable predistortor. The back off $(\mathrm{BO})$ at the nonlinear device can be defined in terms of maximum power output $A^{2}$ as

$$
\mathrm{BO}=10 \log _{10}\left\{\frac{A^{2}}{E\left\{|x|^{2}\right\}}\right\}
$$

where $E\left\{|x|^{2}\right\}$ is the average of the input power to the non-linear device.

\section{RESULTS}

An OFDM system with fixed uplink and down link carriers is simulated. Figure 2 shows the complementary cumulative density function (CCDF) of the PAR of interleaved Zipper signals with $\mathrm{N}=128$. In simulations we assume the half of the 128 subcarriers are assigned for the uplink while the other half is assigned for the down link. PAR reduction of $1.5 \mathrm{~dB}$ is observed at $10^{-3} \mathrm{CCDF}$ compared to the ordinary Zipper signal when a single permutation is used. This reduction increases to 2.5 $\mathrm{dB}$, when 3 permutations are used. We can observe more than $3.5 \mathrm{~dB}$ reduction in PAR when 15 permutations are used. Similar improvements in PAR statistics is observed when $N=256$ and $N=1024$ as shown in Figure 3 and Figure 4.

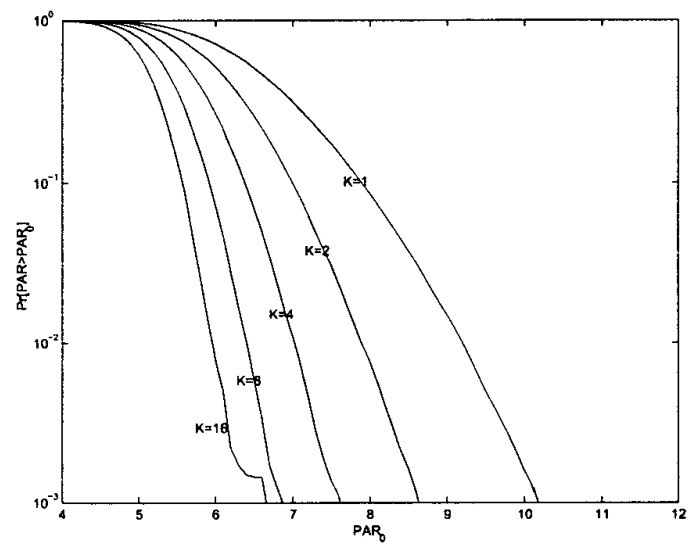

Fig. 3. PAR reduction of a Zipper signal $-N=256$.

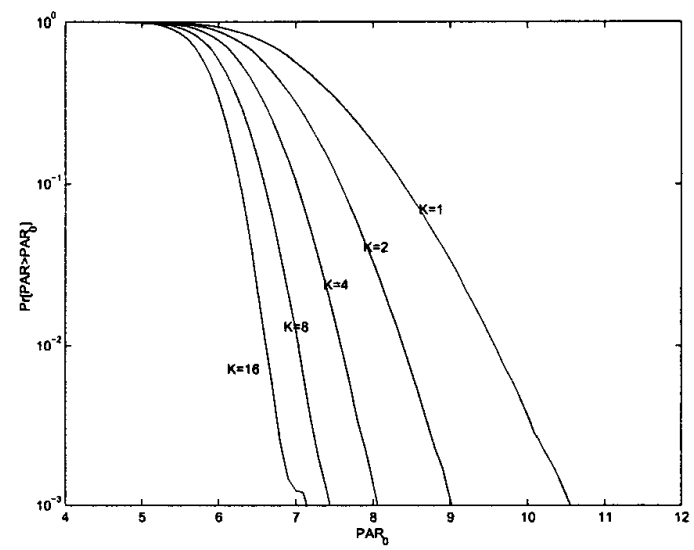

Fig. 4. PAR reduction of a Zipper signal $-N=256$. 


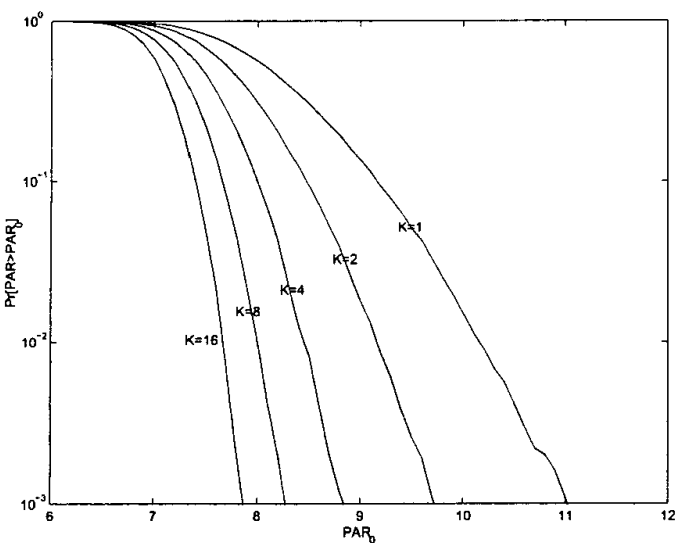

Fig. 5. PAR reduction of a Zipper signal $-N=2048$.

Pulse shaping is a one way of reducing the put of band power of the VDSL Zipper signal [4]. Unfortunately, out of band power may increase again if enough dynamic ranges are not allowed at the transceiver equipment. This regrowth of peaks can be reduced significantly by using a suitable PAR reduction technique. Figure 6 depicts the power spectral density of the Zipper signal when passing through a non-linear device having a soft limiter characteristics defined in Section 3. A relatively high out of band power is observed in ordinary Zipper signals. This gives rise to an increased NEXT. Significant amount of out of band power is reduced when the PAR is controlled. PAR controlled Zipper signal gives out about $-65 \mathrm{dBm}$ out of band power when the back off of the soft limiter is $6 \mathrm{~dB}$. Ordinary Zipper signal has a same amount of out of band power when the back of of the soft limiter is $9 \mathrm{~dB}$. This clearly indicate that we can reduce the dynamic range of the transceivers by $3 \mathrm{~dB}$.

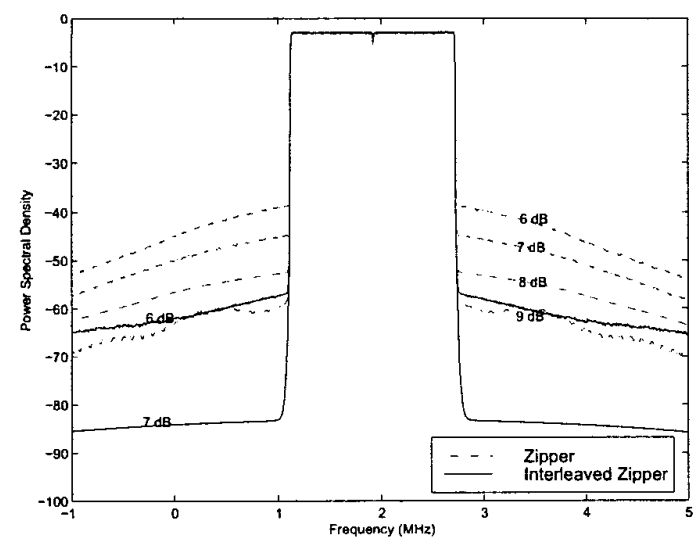

Fig. 6. Power spectral density of a Zipper signal.

\section{CONCLUSIONS}

Peak-to-average power ratio and spectrum of Zipper VDSL signals are investigated in this paper. A data permutation technique is used for PAR reduction. In this approach, $K$ permutations are generated from the input data frame and the permuted frame with the lowest PAR is selected for transmission. By interleaving a data frame, the peaks in the associated OFDM signal can be compressed. The data rate loss of this method is negligible. PAR statistics of different Zipper VDSL signals are presented. PAR can reduced by about $3 \mathrm{~dB}$ in most of the cases with only a moderate increase in complexity. A considerable reduction in out of band power is also observed, which leads to a reduced dynamic ranges for the VDSL transmitter equipment and reduced NEXT.

\section{REFERENCES}

[1] American National Standards Institute, "Very-high-speed digital subscriber lines-systems requirements, draft technical report," ANSI, Austin, TX, Tech REp. T1E1.4/98043R1, 1998.

[2] E. T. S. Institute, "Transmission and multiplexing (TM): access transmission systems on metalic cables: very high speed digital subcriber lines (VDSL) - part 1: Functional requirements," ETSI Tech. Specification TS 101 270-1 VI.I.1 (1998-04), 1998.

[3] F. Sjoberg, M. Isaksson, R. Nilsson, P. Odling, S. K. Wilson, and P. O. Borjesson, "Zipper: A duplex method for VDSL based on DMT," IEEE Trans. Commun., vol. 47, pp. 1245-1252, Aug. 1999.

[4] M. Isaksson and D. Mestdagh, "Pulse shaping with Zipper spectral compatibility and asynchrony," $A N S I$, Austin, $T X$, Tech REp. TIE1.4/98-041, 1998.

[5] E. A. Jones, T. Wilkinson, and S. Barton, "Block coding scheme for reduction of peak-to-mean envelope power ratio of multicarrier transmission schemes," IEE Elect. Lett., vol. 30, pp. 2098-2099, Dec. 1994.

[6] J. Tellado and J. M. Coiff, "Peak power reduction for multicarrier transmission," in IEEE GLOBECOM, (Piscataway, NJ, USA), pp. 219-224, 1998.

[7] C. Tellambura, "Phase optimization criterion for reducing peak-to-average power ratio in OFDM," IEE Elect. Lett., vol. 34, pp. 169-170, Jan. 1998.

[8] A. D. S. Jayalath and C. Tellambura, "Reducing the peakto-average power ratio of an OFDM signal through bit or symbol interleaving," IEE Elect. Lett., vol. 36, pp. 11611163 , June 2000. 